\title{
Estrategias de intervención educativa en el área de inglés en educación primaria para estudiantes con trastorno por déficit de atención e hiperactividad
}

\author{
Educational Intervention Strategies in the English Language Classroom for Students With \\ Attention-Deficit/Hyperactivity Disorder in Primary Education
}

\section{Estratégias de intervenção educacional na área de inglês para estudantes com transtorno de déficit de atenção e hiperatividade na educação primária}

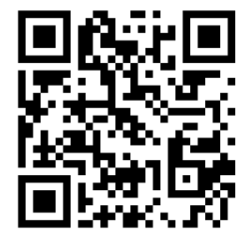

Sandra Moro-Ramos

Universidad de Salamanca

Salamanca, España

smoro@usal.es

https://orcid.org/0000-0002-2351-7059

Recibido • Received • Recebido: 24 / 10 / 2019

Corregido • Revised • Revisado: 28 / 06 / 2021

Aceptado • Accepted • Aprovado: 02 / 08 / 2021

\begin{abstract}
Resumen:
Introducción. El siguiente artículo presenta el análisis que se realizó sobre la pedagogía de una maestra de lengua extranjera (inglés) en un colegio de educación primaria. Objetivo. El objetivo de esta actuación es analizar la pedagogía de la especialista en lengua extranjera (inglés); concretamente, examinar las medidas llevadas a cabo con el alumnado con trastorno por déficit de atención e hiperactividad (TDAH). Se pretende plantear un modelo realista en el paradigma de la enseñanza y el aprendizaje de la lengua anglosajona como segunda lengua con escolares con TDAH. Metodología. A través de la técnica de investigación documental, se revisará la práctica educativa, se analizarán y pondrán en práctica diversas metodologías y se modificará la praxis educativa para añadir los ajustes de intervención necesarios para que todo el alumnado, con TDAH o sin este, aprenda inglés como ESL (del inglés English as a Second Language). Resultados. A partir de este análisis, se mostrarán una serie de estrategias para trabajar el área de inglés con escolares con TDAH. Discusión. Partiendo de que este es un trastorno neurobiológico en el que la repercusión que tiene en cada persona es distinta y las áreas en las que pueden presentar problemas difieren de unas a otras, se proponen diversas actividades y tácticas basadas en el análisis del discurso para dotar a estas personas de las destrezas necesarias para la adquisición del inglés como segundo idioma. Asimismo, en el presente artículo se muestran dos modelos para el entrenamiento cognitivo de niños y niñas con hiperactividad o sin esta para actividades orales y escritas en inglés como L2.
\end{abstract}

Palabras claves: Análisis del discurso; dificultades de aprendizaje; enseñanza de idiomas; inglés, lengua extranjera; TDAH. 
http://doi.org/10.15359/ree.25-3.20

http://www.una.ac.cr/educare

educare@una.ac.cr

\begin{abstract}
:
Introduction. This article analyzes the pedagogy of an ESL teacher in a primary school. Objective. This investigation aims to evaluate the instruction of the ESL teacher, more specifically to analyze the measures implemented with attention-deficit/hyperactivity disorder (ADHD) learners. All these ideas outline a realistic model in the paradigm of teaching and learning English as a second language with ADHD students. Methods. Under a desk research scope, the teacher's instruction is reviewed, several methodologies are analyzed and put into practice, and the educational practice is modified to adjust the intervention procedures to ensure that all pupils, with or without ADHD, learn ESL. Results. As a result of this study, several techniques are recommended to teach ESL to ADHD students. Discussion. Based on the fact that ADHD is a neurobiological disorder where the impact in every person diverges and the possible problematic areas differ from one to another, several activities and techniques are proposed to provide these individuals with the skills they need to acquire English as a second language. Additionally, this article also proposes two cognitive training self-instruction models for children, with and without ADHD, for oral and written activities in ESL.
\end{abstract}

Keywords: Discourse analysis; learning disabilities; teaching languages; English; foreign language; ADHD.

\begin{abstract}
Resumo:
Introdução. O seguinte artigo apresenta a análise realizada sobre a pedagogia de uma professora de língua estrangeira (inglês) em um colégio de Educação Primária. Objetivo. O objetivo desta atuação é analisar a pedagogia da especialista em língua estrangeira (inglês), mas concretamente, examinar as medidas realizadas a estudante com TDAH (Transtorno de Déficit de Atenção e Hiperatividade). Pretende-se planejar um modelo realista no paradigma do ensino e a aprendizagem da língua anglosaxônica como segunda língua a estudantes com TDAH. Metodologia. Através da técnica de pesquisa documental, revisar-se-á a prática educativa; várias metodologias serão analisadas e colocadas em prática, e modificar-se-á a práxis educacional para acrescentar os ajustes de intervenção necessários para que o grupo de estudantes com ou sem TDAH, aprendam inglês como ESL (do inglês "English as a Second Language"). Resultados. A partir desta análise será mostrada uma série de estratégias para trabalhar a área de inglês com estudantes com TDAH. Conclusão. Partindo de que este é um transtorno neurobiológico no qual a repercussão que tem em cada pessoa é diferente e as áreas nas quais podem apresentar problemas diferem de uns a outros, propõem diversas atividades e táticas baseadas na análise do discurso para dotar a estas pessoas com as destrezas necessárias para a aquisição do inglês como segundo idioma. Da mesma forma, no presente artigo mostram-se dois modelos para o treinamento cognitivo de estudantes com ou sem hiperatividade para atividades orais e escritas em inglês como L2.
\end{abstract}

Palavras-chave: Análise do discurso; dificuldades de aprendizagem; ensino de idiomas; inglês língua estrangeira; TDAH.

\title{
Introducción
}

El trastorno por déficit de atención con hiperactividad o sin esta (TDAH) es una alteración neurobiológica que afecta a un 3,7\% de infantes, lo que equivale a un sujeto o dos escolares por cada 25 estudiantes (Quintero Gutiérrez del Álamo y García Campos, 2019). 
http://doi.org/10.15359/ree.25-3.20

EI TDAH es un trastorno neurobiológico que se manifiesta por una hiperactividad, impulsividad y autocontrol inapropiado, así como por las dificultades para mantener la atención (Barkley, 1990). Por lo tanto, presenta alteraciones en la atención y control de los impulsos o conductas, así como un exceso de movimientos.

Este trastorno se divide en tres tipos, según los síntomas predominantes del DSM-V (American Psychiatric Association, 2013):

- TDAH de tipo inatento: Predominan los síntomas relativos al déficit de atención.

- TDAH de tipo hiperactivo/impulsivo: Predominan los síntomas relativos a la hiperactividad-impulsividad.

- TDAH de tipo combinado: Presentan síntomas combinados de los dos tipos anteriores.

Por otro lado, el TDAH es un desorden con un elevado grado de morbilidad asociada con otros trastornos (dificultades de aprendizaje, trastorno de conducta, trastorno oposicionistadesafiante, ansiedad, trastornos emocionales, tics, etc.).

Uno de los principios que aparecen en el Capítulo 1 de la LOMCE (Jefatura del Estado, 2013) establece lo siguiente:

Artículo 1. Principios:

b) La equidad, que garantice la igualdad de oportunidades para el pleno desarrollo de la personalidad a través de la educación, la inclusión educativa, la igualdad de derechos y oportunidades que ayuden a superar cualquier discriminación y la accesibilidad universal a la educación, y que actúe como elemento compensador de las desigualdades personales, culturales, económicas y sociales, con especial atención a las que se deriven de cualquier tipo de discapacidad. (p. 11)

Si evaluamos lo estipulado por ley y lo comparamos con los resultados educativos que tienen algunos alumnos y alumnas con TDAH, llegamos a la consideración de que los cuerpos docentes debemos mejorar el trabajo con las personas que padecen este trastorno, para que tengan las mismas oportunidades y éxito que las demás.

La escasez de estrategias de intervención educativa para enseñar una lengua extranjera como L2 con alumnado conTDA/TDAH provocaron la motivación para trabajar diversos aspectos y metodologías y poder dar a todo el alumnado la misma oportunidad de aprender inglés como segunda lengua y, por consiguiente, la elaboración de la investigación a la que pertenece este artículo. La pretensión del presente estudio era evaluar la práctica educativa de una maestra de lengua extranjera (inglés) en un colegio de Educación Primaria para, posteriormente, trazar y desarrollar una nueva programación didáctica con ajustes de intervención para que todos los 
http://doi.org/10.15359/ree.25-3.20

http://www.una.ac.cr/educare

educare@una.ac.cr

niños y niñas, incluyendo a estudiantes con TDAH, aprendieran inglés como segunda lengua. Durante este proceso se pusieron en práctica múltiples actividades para ejercitar las cuatro habilidades básicas de una lengua, con diversas metodologías se crearon una serie de estrategias para trabajar inglés como segunda lengua y se diseñaron dos esquemas de autoinstrucciones basados en planteamientos de Orjales Yillar (2005) para el entrenamiento cognitivo de niños y niñas con hiperactividad o sin esta, uno para actividades orales y otro para actividades escritas.

\section{Marco teórico}

\section{Análisis del discurso}

El análisis del discurso es una disciplina que estudia el lenguaje en uso, es decir, analiza la lengua y los contextos en los que esta se emplea, su uso real. Nace en los años 60 y principios de los 70 a partir de otras disciplinas, como lingüística, semiótica, pragmática, psicología, antropología o sociología, entre otras (McCarthy, 1991).

Como indica McCarthy (1991), Zellig Harris fue la primera persona que introdujo la noción de análisis del discurso en la comunidad científica, definiéndolo como un sistema de estudio conectado al habla (o a la escritura). Son muchos los autores y autoras que se han dedicado a realizar dicho análisis, entre ellos está Cook (1989), quien considera que el análisis del discurso es un ámbito en desarrollo que interconexiona la pronunciación, la gramática y el vocabulario con el objetivo de conseguir una comunicación eficiente.

El análisis del discurso se interesa en el uso de la lengua. Teniendo en cuenta que el profesorado de lenguas no solo emplea una lengua como vehículo de transmisión, sino que instruye la propia lengua en sí, son muchos los condicionantes a considerar a la hora de impartir docencia, como, por ejemplo, los materiales que se van a emplear; las situaciones que se dan en cada aula, en cada momento de la enseñanza; el alumnado y sus características individuales; o los recursos materiales que poseen.

Cook (1989) y McCarthy (1991), dos autores consagrados en el ámbito del discurso, consideran primordial tener en consideración el lenguaje que se emplea día a día en la enseñanza de lenguas, así como las diferencias culturales que coexisten e influyen en la producción e interpretación del discurso. Sin llegar a considerar al análisis del discurso como un método de docencia, Cook (1989) considera que la clase de lengua extranjera es una de las mejores fuentes de análisis para el análisis del discurso, así como el área más precisa para estudiar su teoría.

El discurso tiene también un impacto sobre las personas, tanto a nivel lingüístico como personal, puesto que las decisiones lingüísticas que se toman en los procesos de comunicación, el registro y los ejemplos empleados, así como la entonación o las expresiones verbales y físicas que el profesorado emplea en sus clases juegan un papel fundamental sobre sus estudiantes 
http://doi.org/10.15359/ree.25-3.20

(Vetter et al., 2018). El objetivo de emplear el análisis del discurso en clase, tal y como indica Rymes (2016), es comprender los registros comunicativos de cada individuo para posibilitar y fomentar diálogos y aprendizajes inclusivos.

\section{Trastorno por déficit de atención (con hiperactividad o sin esta)}

EI TDAH es un trastorno caracterizado por la impulsividad y la hiperactividad. Se usa con los acrónimos de TDA (trastorno por déficit de atención) o TDAH (trastorno por déficit de atención e hiperactividad), aunque el término TDAH se emplea para hacer alusión a los tres tipos existentes.

Tal y como indica el Dr. Russel A. Barkley (Barkley, 2013), el TDAH no es solo un trastorno relacionado con la falta de control de los impulsos o de la atención, sino que se trata de una disfunción que afecta también al control del tiempo o a las consecuencias de nuestros actos, puesto que el TDAH puede afectar nuestras funciones ejecutivas. Las funciones ejecutivas son las habilidades cognitivas que nos ayudan a organizar, planificar y controlar nuestro cerebro y, consecuentemente, la conducta y el habla. Las personas con TDAH suelen ser más impulsivas y descuidadas que el resto, y son estos rasgos la causa de muchos de sus problemas, no solo académicos, sino familiares y personales también. Podría decirse que muchos de sus obstáculos en la vida tienen su raíz en las deficiencias de las funciones ejecutivas, como son la memoria de trabajo, el habla autodirigida, el control de la motivación y la planificación y organización (Cooper-Kahn y Foster, 2013). Es importante señalar que no todas las personas con TDAH tienen disfunciones ejecutivas.

Anteriormente hemos referido los distintos tipos de TDAH. En función de la predominancia de los síntomas, estos tendrán más o menos dificultades en diferentes áreas.

\section{TDAH de tipo inatento}

Las dificultades académicas en el área de lenguas relacionadas con la inatención se encuentran fundamentalmente en las siguientes habilidades (Rief, 2005):

- Lectura: Con frecuencia tienen dificultades para seguir las lecturas; les cuesta mantener la atención sobre el texto, sobre todo si este es extenso o difícil; olvidan con frecuencia lo que han leído.

- Escritura: Tienen dificultad para planificar y organizar las tareas; con frecuencia cometen errores por descuido en las tareas; no suelen terminar las tareas o las hacen de forma escasa; no realizan el trabajo con precisión; cometen errores ortográficos y de puntuación, pues les aburre prestar atención a los detalles. 
http://doi.org/10.15359/ree.25-3.20

http://www.una.ac.cr/educare

educare@una.ac.cr

\section{TDAH de tipo hiperactivo/impulsivo}

Las dificultades académicas en el área de lenguas relacionadas con la inatención se encuentran fundamentalmente en los siguientes comportamientos:

- Con frecuencia se levantan cuando no deben; no pueden permanecer quietos, corren, saltan, juegan con objetos o hacen ruidos cuando no es apropiado; hablan excesivamente y tienen problemas para respetar turnos; responden antes de que se acaben de formular las preguntas; necesitan mover las manos olos pies en exceso; tienen dificultades para respetar las normas de clase; interrumpen o molestan a compañeros y compañeras; se aburren con facilidad; son impacientes; tienen dificultades para realizar tareas o participar en juegos de forma tranquila y silenciosa.

Brouwer Konyndyk (2011) muestra en su manual Foreign Languages for everyone. How I learned to teach second languages to students with learning disabilities, cómo el alumnado con dificultades de aprendizaje puede aprender una lengua extranjera; considera, como requisito indispensable para lograrlo, la adaptación de las estrategias de enseñanza a las necesidades de todo el estudiantado. Por lo tanto, es fundamental que los profesores y las profesoras de ESL (English as a Second Language) tengan en cuenta las características y necesidades individuales de todos los sujetos aprendices que hay en las aulas, e introduzcan algunos cambios en el trabajo que realizan con los individuos con TDAH, para que alcancen los objetivos y tengan las mismas oportunidades que los demás.

\section{Metodología}

El presente artículo forma parte de un estudio más amplio, que pretende dar respuesta a las necesidades que estudiantes con TDAH poseen a la hora de aprender inglés como segunda lengua. Habiendo numerosas investigaciones relativas a la enseñanza de asignaturas troncales con alumnado con TDAH, como lengua y matemáticas, descubrimos que no había muchos estudios que abordaran las estrategias necesarias para trabajar con dichas poblaciones en la enseñanza-aprendizaje del inglés como segunda lengua. Por ello, consideramos fundamental realizar una investigación-acción, para poder dar respuesta a las necesidades de nuestro alumnado. Para realizar este estudio seguimos los siguientes pasos de forma cíclica durante varios cursos: planificación, acción, observación y reflexión.

La recolección de información se realizó a través de la técnica de investigación documental y realizando cursos especializados en TDAH y en metodología de idiomas. Se recopilaron y analizaron diversos libros y artículos relativos al TDAH, al análisis del discurso y a la enseñanza de lenguas. En varias aulas de Educación Primaria se trabajó de forma inclusiva con estudiantes con TDAH. El análisis se realizó durante tres años, tiempo en el que algunas personas del alumnado de las diversas aulas padecían el trastorno. 
Nuestro principal objetivo se basaba en cambiar la actividad educativa, adquiriendo una formación específica para trabajar con alumnado con TDAH y mejorar la pedagogía, pretendiendo obtener las herramientas adecuadas para darle la atención educativa necesaria, tanto para quienes poseen un trastorno por déficit de atención con hiperactividad o sin esta, como para quienes no lo poseen. Con esta investigación, a partir de las estrategias y las actividades propuestas, así como con la práctica de los dos esquemas de autoinstrucciones creados, se pretendía ayudar a otro profesorado de ESL con alumnos y alumnas con TDAH.

Como procedimientos, se crearon una serie de estrategias sobre cómo enseñar inglés como lengua extranjera al alumnado con TDAH y se diseñaron dos esquemas de autoinstrucciones para el entrenamiento cognitivo de niños y niñas entre 9 y 11 años con hiperactividad o sin esta, uno para actividades escritas (Figura 2) y otro para actividades orales (Figura 4), a partir de la propuesta de Orjales Yillar (2005). Con ello, se pretendía modificar la conducta del alumnado con TDAH, aumentar su autoestima y, consecuentemente, lograr una mejora en los conocimientos del área de inglés.

En cuanto a las fases, se distinguen:

- Fase de evaluación: Como el propio nombre indica, se realizó una evaluación de la práctica educativa llevada a cabo, sopesando los pros y los contras de las actividades hasta el momento realizadas, para poder determinar cuáles se debían o se podían cambiar para poder desarrollar y emplear la labor docente al máximo en el periodo de 60 minutos que duran las clases.

- Fase de observación: Un paso muy importante fue observar las aulas en las que se iba a trabajar, para tener en cuenta el material y el espacio del que se iba a disponer para desarrollar las sesiones. Asimismo, era primordial observar a los distintos niños y niñas y ver cómo trabajaban y cómo se desenvolvían, tanto a nivel individual como grupal para, teniendo todo ello en cuenta, programar actividades que beneficiaran a todo el grupo.

- Fase deliberativa y motivadora: Se realizaron cursos de especialización de TDAH y de metodología de idiomas y se leyeron y consultaron libros y artículos sobre análisis del discurso, enseñanza de segundas lenguas y TDAH.

- Fase demostrativa:Se aplicaron varias metodologías para trabajar las cuatro habilidades básicas de la lengua inglesa, alternando el método clásico, con el métodoTPR (del inglés total Physical Response), con el método silencioso, con la instrucción basada en tareas, entre otras técnicas. Para poder realizar dichos cambios, se incluyeron actividades y juegos diversos, como se explicará a continuación y algunos ejercicios de relajación (inspirar por la nariz y expirar por la boca, visualización de imágenes relajantes a través de palabras suaves, etc.).

- Fase de evaluación: Finalmente se realizó una evaluación de la práctica educativa y un análisis de los cambios y resultados obtenidos en el alumnado. 
http://doi.org/10.15359/ree.25-3.20

http://www.una.ac.cr/educare

educare@una.ac.cr

\section{Actividades}

Con la pretensión de trabajar las cuatro habilidades lingüísticas del inglés con el alumnado con TDAH y sin este, para que todo el grupo fuera capaz de adquirir la lengua inglesa, a lo largo del presente estudio se han realizado actividades de comprensión y expresión escrita y de comprensión y expresión oral. Las actividades se han realizado con alumnos con TDAH y sin este, puesto que el estudio se ha llevado a cabo en aulas inclusivas en las que la atención individualizada se realiza dentro del aula. Para realizar las actividades se han consultado y puesto en práctica varios libros, destacan el de Monschein (2008) y el de Rinvolucri y Davis (1995). Para las actividades de TPR (Total Physical Response) ha sido de gran ayuda el manual de Lengel y Kuczala (2010).

A continuación, se exponen algunos ejemplos de las actividades en las que se ejercita en análisis del discurso las cuales se han realizado a lo largo de este estudio:

\section{Actividades de ESL en las que se ejercita el análisis del discurso}

Ivanov (2009) explica algunas de las recomendaciones que Lezberg y Hilferty (1978) ofrecen al profesorado que trabaja la comprensión escrita como discurso en la clase. Entre ellas destacan las actividades de scanning y skimming, actividades que pueden realizarse en varios momentos: antes, durante y después de la lectura. Las actividades de "skimming" consisten en hacer una lectura rápida, buscando ideas o frases y tienen como objetivo reconocer rápidamente las principales ideas de un texto. Por el contrario, las actividades de scanning se basan en encontrar la información concreta que nos solicitan. Su objetivo es realizar una lectura analítica, más atenta.

En cuanto a las actividades previas a la lectura, se han tenido en cuenta los conocimientos y experiencias de los lectores y lectoras, empleados para vaticinar el contenido de la lectura. Para ello debían fijarse en el título y en las ilustraciones, cuando las había. Estas actividades preliminares a la lectura a través de inferencias de imágenes también se han aprovechado para realizar actividades de "speaking" (del inglés "hablar"). A partir de las fotografías y dibujos que acompañaban a los textos, se pedía a al grupo de discentes que explicaran las imágenes, trabajando así la habilidad de hablar (o "speaking" en inglés) y después se les preguntaba por la relación que consideraban tenían con el texto. A lo largo de la lectura se han realizado actividades para constatar que al leer estaban entendiendo el contenido, es decir, actividades de comprensión lectora, bien preguntando por algún dato en concreto, pidiendo un resumen o dando algún dato erróneo para que los niños y las niñas lo corrigieran. Una vez finalizada la lectura, se han realizado diversos tipos de actividades, en algunas ocasiones se han realizado actividades de vocabulario, como búsqueda de sinónimos, así como responder a preguntas,

actividad que no solo sirve para ahondar en la comprensión lectora, sino también para practicar 
la habilidad de escritura de una lengua. En ocasiones se han propuesto actividades de escritura a partir de lectura, pidiendo al estudiantado que cambiara el final de una historia y, si se disponía de menos tiempo o el ambiente de la clase lo permitía, se ha realizado de forma grupal e incluso de forma oral. El único inconveniente de hacerlo de forma oral es que siempre va a haber estudiantes que, aunque se beneficien de escuchar a sus pares, no participan activamente a no ser que se les indique expresamente. Aunque puedan parecer actividades sencillas, son ejercicios que requieren de una capacidad de concentración y organización mental que a veces resulta complicada para algunas personas con TDAH. Si a esto le sumamos las carencias de vocabulario y las dificultades de comprensión gramaticales que una parte del grupo posee, puede resultar difícil que discentes con TDAH puedan realizar este clase de actividades sin ningún tipo de ayuda. Por lo tanto, la práctica de estas actividades les ayuda a adquirir las estrategias adecuadas para poder realizarlas de forma autónoma.

\section{Actividad logopédica-fonológica de ESL}

A continuación, presentamos una actividad logopédica-fonológica que puede ayudarnos a mejorar la expresión oral en inglés. Juego As Light as a Feather: Esta sencilla propuesta presentada por Monschein (2008), en el libro The 50 best games for speech \& language development, es una actividad que se puede plantear al principio de la clase como actividad de calentamiento, al final de la clase como actividad de relajación o se puede realizar al trabajar los fonemas / $v$ / y / u: /.

Para realizar esta actividad los niños y niñas necesitan una pluma, cuanto más ligera mejor. El juego consiste en mantener el objeto en el aire soplando, resulta ganador o ganadora aquel o aquella que sea capaz de mantenerlo en el aire por más tiempo.

Una vez realizada la prueba, se practicaron los fonemas / $v / \mathrm{y} / \mathrm{u}$ : / y pronunciamos algunas palabras que contienen este sonido como, por ejemplo: book, cook, look, soon, spoon.

Gran cantidad de aprendices con TDAH tienen problemas para organizar sus ideas y, consecuentemente, el discurso. Los problemas de autoestima que poseen, sumados a las dificultades que acabamos de mencionar, les limitan en muchas ocasiones a la hora de poner en práctica las habilidades de expresión oral. La realización de ejercicios logopédicos como el que acabamos de exponer les ayuda a pronunciar mejor, lo que mejora la confianza que poseen en sí, lo cual repercute positivamente en las producciones orales del alumnado con TDAH y sin esta.

\section{Actividad de TPR}

Juego Emperor, How far May I Travel?: Este juego fue diseñado por Monschein (2008) para desarrollar las habilidades de comprensión oral. Las personas aprendices se debían poner a un lado de la clase y la profesora, en este caso, o uno de los compañeros o compañeras que hiciera de "emperador" se debía situar al otro lado. La clase preguntaba: Emperor, how far may I 
http://doi.org/10.15359/ree.25-3.20

http://www.una.ac.cr/educare

educare@una.ac.cr

travel? (emperador, ¿hasta dónde puedo viajar?). El emperador, en su respuesta, podía emplear nombres propios, nombres de países, etc. En realidad, es una actividad que acepta multitud de variables en la respuesta del emperador, dependiendo de lo que se quiera practicar. El número de sílabas de la respuesta determina el número de pasos que los demás compañeros pueden dar. La primera persona en llegar al lado opuesto de la clase gana. En las prácticas realizadas, los niños y las niñas que llegaban en primer lugar se convertían en emperadores o emperadoras.

Es una actividad que se puede emplear como calentamiento o como actividad de final de sesión, aunque tras su puesta en práctica se recomienda realizarla al final, puesto que se genera mucho revuelo y alboroto al realizarla, ya que suele gustar mucho e implica el movimiento de todo el grupo-clase. Practicamos el vocabulario estudiado y los verbos, reforzando así los conocimientos trabajados con el grupo de discentes durante el tema y los verbos, contenido que suele ser difícil de adquirir por muchos individuos con TDAH. Esto se debe a las dificultades que estas personas suelen poseer con las funciones ejecutivas. Las actividades de TPR permiten el movimiento físico, lo que resulta de gran agrado para algunos alumnos y alumnas con TDAH, pues favorece su concentración e implicación y hace que algunos rasgos característicos de su impulsividad pasen inadvertidos. Este tipo de actividades, a su vez, reducen el nivel de estrés que se posee a la hora de adquirir un segundo idioma.

\section{Estrategias de intervención en el área de inglés para alumnado con TDA-TDAH}

Puesto que adquirimos una lengua con el propósito de comunicarnos, en nuestro caso la lengua inglesa, hemos elegido el análisis del discurso como marco teórico, ya que es una disciplina que estudia el lenguaje en uso.

Para poder plantear un modelo realista en el paradigma de la enseñanza y el aprendizaje de la lengua anglosajona como segunda lengua con alumnado con TDAH, debemos conocer el trastorno y las características de cada persona con TDAH en concreto, para poder considerar qué tipo de problemas debemos tener en cuenta para poder trabajar específicamente en los aspectos que presentan dificultades, primeramente en la L1 (lengua materna, en este caso lengua española) y, extensivamente, en la L2 (segunda lengua, en nuestro caso, inglés) y, de este modo, conseguir emplear el inglés para comunicarnos y socializarnos.

Durante los tres años que se duró el presente estudio, más de diez aprendices tenían TDAH, siendo dispares los problemas específicos del lenguaje que tenían. Partiendo de la base de que no todo el alumnado con TDAH tiene problemas con el lenguaje, y que cada individuo presenta unas características y necesidades particulares, presentamos de forma genérica los problemas que podemos encontrar a la hora de trabajar con personas con TDAH en el área de lengua: 
http://doi.org/10.15359/ree.25-3.20

- Sintaxis: Problemas con el uso o comprensión de los componentes de la oración.

- Semántica: Problemas con la organización y el significado de las palabras.

- Problemas de comprensión (tanto del lenguaje oral como del lenguaje escrito).

- Pobreza de vocabulario.

- Pragmático-discursiva: Problemas de expresión y de relaciones. Hablan en exceso, pero tienen problemas para mantener una conversación ordenada y coherente o para expresar sentimientos.

- Metalingüística: Problemas con los giros y el sentido figurativo del lenguaje.

A lo largo de este artículo abordaremos cómo trabajar con las personas con TDAH y les daremos herramientas y estrategias para paliar dichos problemas.

Lo que es evidente es que los grupos de escolares que padecen TDAH pueden dominar perfectamente un segundo idioma, lo único que hace falta es que el profesorado sepa cuáles son sus necesidades para ayudarles a conseguir su meta y respete sus ritmos de aprendizaje, que muchas veces son más lentos que el de el resto de sus compañeros o compañeras.

A continuación, mostraremos cómo trabajar en el aula el área de inglés con estos niños y niñas, a través de una serie de estrategias de intervención, abordando las dificultades de aprendizaje que se nos pueden presentar en el desarrollo de las cuatro habilidades básicas de una lengua (comprensión y expresión escrita y comprensión y expresión oral).

\section{Dificultades en la lectura}

- Realizar fichas de comprensión lectora acordes a su nivel.

- Habituar al alumnado a buscar en el diccionario las palabras que desconoce.

- Buscar textos motivantes para la niñez.

- Seguir la lectura con un lápiz o "señalador" para no perderse.

- Practicar actividades de "skimming" y"scanning".

- Hacer preguntas para asegurarnos que comprenden los textos.

- Hacer juegos con acrónimos, metáforas y oxímoron. 
http://doi.org/10.15359/ree.25-3.20

http://www.una.ac.cr/educare

educare@una.ac.cr

\section{Dificultades en la escritura}

- Plantear crucigramas, unscramble, stairs (juego de acrónimos) y otros ejercicios de palabras.

- Jugar al ahorcado.

- Diseñar actividades con el diccionario como, por ejemplo: nombrar pictogramas (Figura 1).

Figura 1: Pictograma.

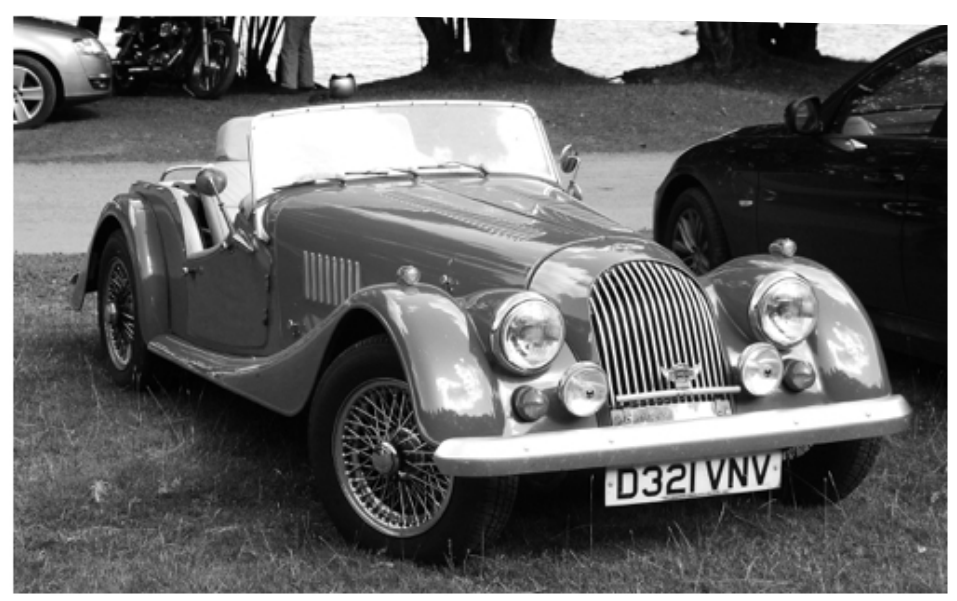

Nota: Fotografía propiedad de la autora Sandra Moro Ramos.

- Ayudar al aprendiz a comprender los componentes estructurales de la gramática, ofreciendo el tiempo suficiente para practicarlos, recordándolos frecuentemente y premiando el esfuerzo realizado, independientemente de que, por su edad, ya debiera haberlas adquirido.

- Permitir el uso del ordenador para escribir o un procesador de textos.

- Respetar el ritmo de aprendizaje del niño o niña con TDA/TDAH.

- Poner en práctica el esquema de autoinstrucciones para el entrenamiento cognitivo de niños y niñas con hiperactividad o sin esta para actividades escritas (modificado a partir de la propuesta de Orjales Yillar (2005): 
http://doi.org/10.15359/ree.25-3.20

http://www.una.ac.cr/educare educare@una.ac.cr

Figura 2: Esquema de autoinstrucciones para el entrenamiento cognitivo de niños y niñas con o sin hiperactividad para actividades escritas, modificado a partir de la propuesta de Orjales Yillar (2005)
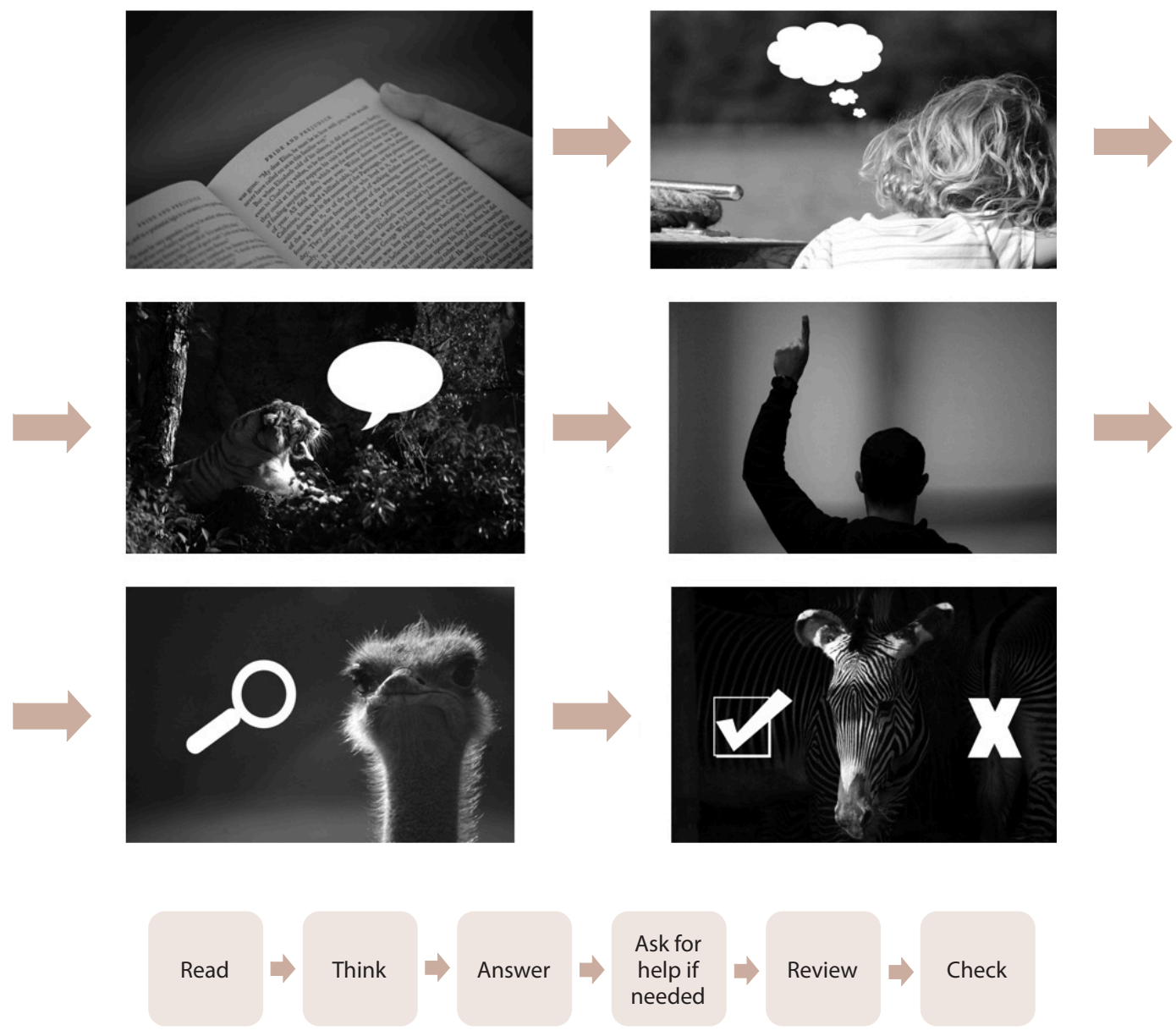

Nota: Fotografía propiedad de la autora Sandra Moro Ramos.

\section{Dificultades en la comprensión oral}

- Repetir las producciones orales si es necesario para que los niños y las comprendan.

- Hacer uso de apoyos visuales y gestos.

- Dar instrucciones cortas y sencillas.

- Buscar gestos de complicidad con el niño o la niña con TDA/TDAH para saber que está comprendiendo el mensaje. 
http://doi.org/10.15359/ree.25-3.20

http://www.una.ac.cr/educare

educare@una.ac.cr

- Variar el tono de voz y acompañar la emisión de mensajes y preguntas con gestos.

- Hacer juegos con acrónimos, metáforas y oxímoron.

- Hacer uso de la "técnica sándwich" (inglés-español-inglés, tal y como se muestra en la Figura 3) cuando el alumno o la alumna no es capaz de captar el mensaje general o específico, a pesar de emplear gestos y apoyos visuales.

Figura 3: Esquema de la "técnica de sándwich" (inglés-español-inglés)

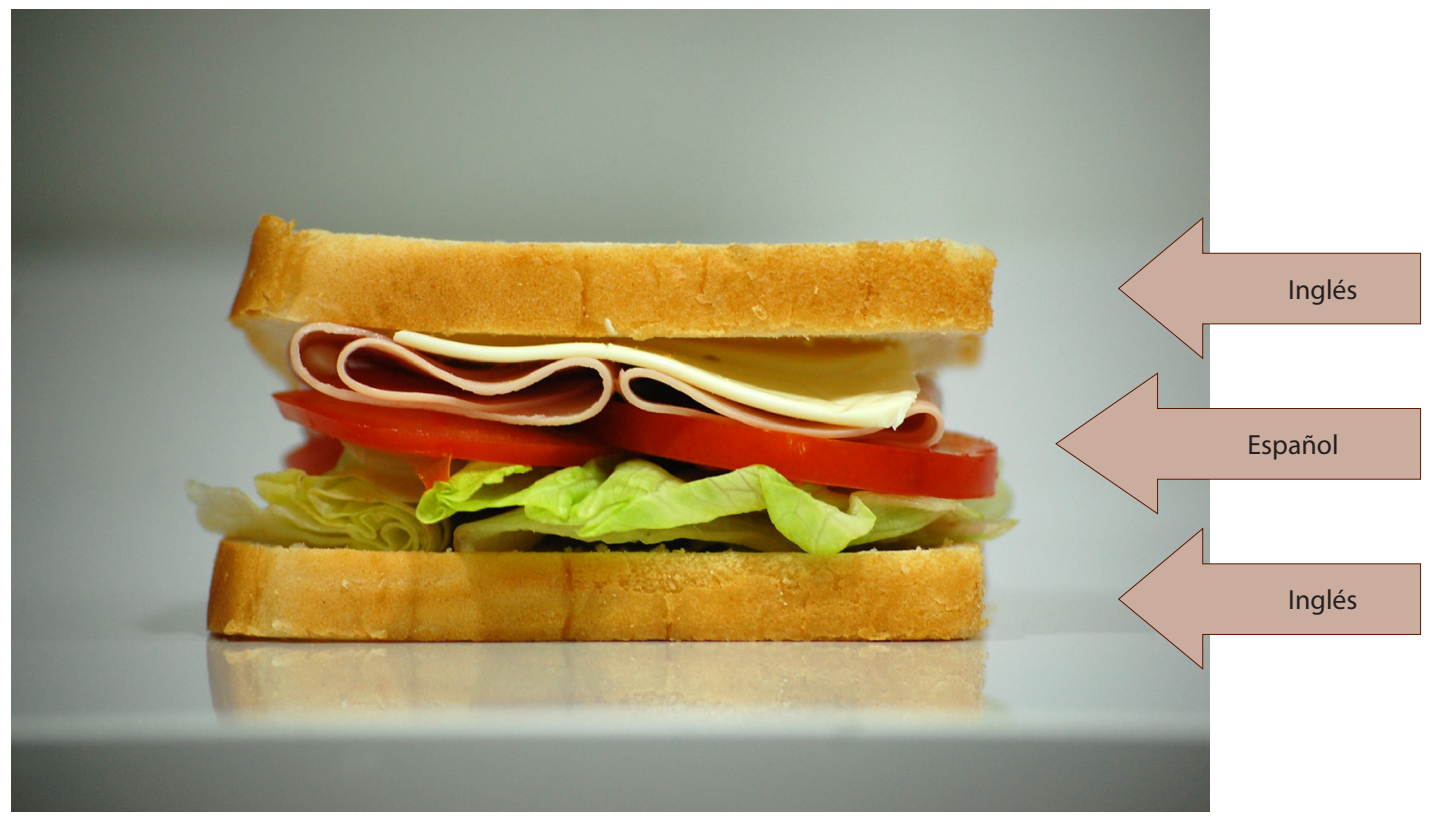

Nota: Fotografía propiedad de la autora Sandra Moro Ramos.

- Emplear el sentido del humor o hacer uso de anécdotas para mantener la atención del niño y la niña.

- Ayudar al estudiantado a comprender el contexto lingüístico para minimizar los problemas pragmáticos que pueda poseer a través del análisis del discurso y del refuerzo de su autoestima.

- Crear imágenes mentales. Un modo de que el alumnado mantenga la conexión es pedirle que genere imágenes mentales de los mensajes que escucha.

- Poner en práctica el esquema de autoinstrucciones para el entrenamiento cognitivo de niños y niñas con hiperactividad o sin esta para actividades orales (modificado a partir de la propuesta de Orjales Yillar (2005): 
http://doi.org/10.15359/ree.25-3.20 http://www.una.ac.cr/educare educare@una.ac.cr

Figura 4: Esquema de autoinstrucciones para el entrenamiento cognitivo de niños y niñas con o sin hiperactividad para actividades orales, modificado a partir de la propuesta de Orjales Yillar (2005)
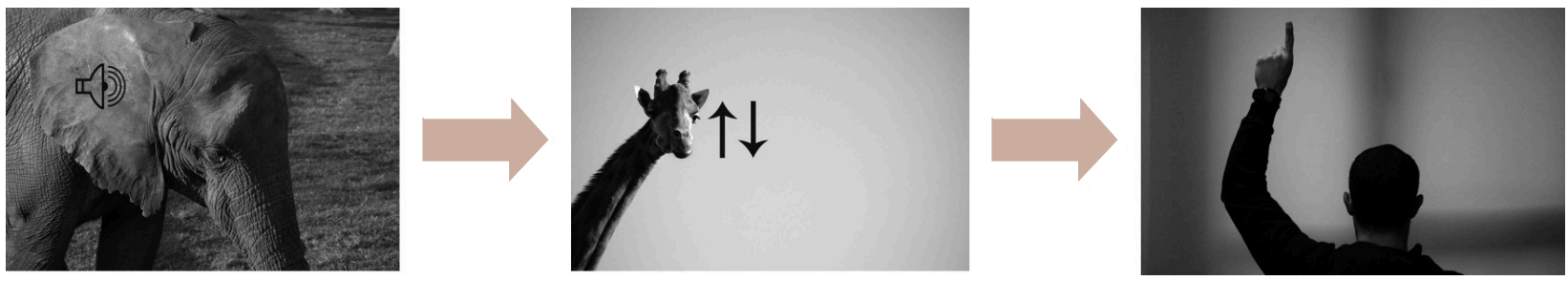

Listen
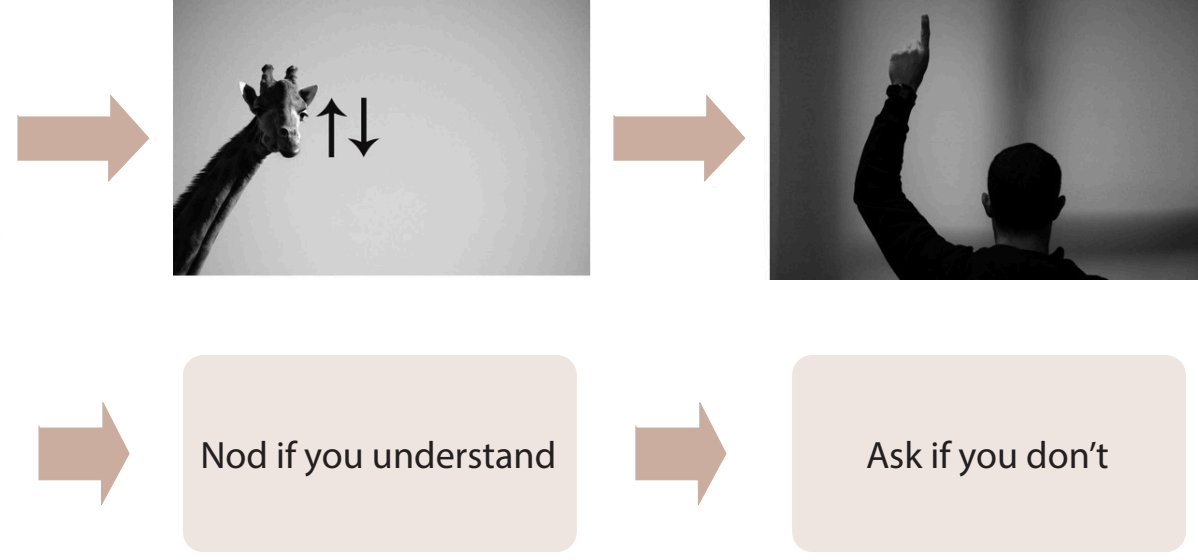

Nota: Fotografía propiedad de la autora Sandra Moro Ramos.

\section{Dificultades en la producción oral}

- Animar a la niñez a expresarse en inglés.

- Permitirles hablar en "spanglish" si no son capaces de emitir el mensaje completo en el lenguaje anglosajón, y ayudarles diciéndoles las palabras que les faltan en inglés.

- Darles tiempo para responder, transmitiéndoles calma y dejándoles un tiempo para que preparen el mensaje si lo necesitan.

- Ayudar a la niñez con TDA/TDAH a comprender el contexto lingüístico y los elementos lingüístico-pragmáticos.

- Premiar el comportamiento correcto, como por ejemplo, no interrumpir o respetar los turnos de palabra.

- Retroalimentar al alumnado: Alabar los progresos y corregir los errores con mensajes positivos. Es importante que la niñez con TDA/TDAH sienta que está progresando y que puede conseguir los objetivos que se proponen.

\section{Resultados, análisis y discusión}

Tal y como hemos señalado anteriormente, las investigaciones relativas al desarrollo académico de alumnado con TDAH son numerosas, pero los estudios relativos a la enseñanza del inglés como segunda lengua con estudiantes con TDAH eran escasos al comienzo de la 
http://doi.org/10.15359/ree.25-3.20

http://www.una.ac.cr/educare

educare@una.ac.cr

investigación. Por ello, nuestro principal objetivo consistía en obtener las herramientas adecuadas para atender a todos nuestros alumnos y alumnas con TDAH y sin esta durante las clases de inglés.

Para conseguirlo, realizamos múltiples actividades durante varios años, poniendo en practica varias metodologías en aulas de Educación Primaria para tratar de satisfacer las necesidades que el alumnado con TDA/TDAH tienen a la hora de aprender inglés como segunda lengua. Del mismo modo, pusimos en práctica diversas estrategias siguiendo las recomendaciones de Barkley (2013) y Rief (2005), estableciendo un sistema de intervención que nos permitió a trabajar obteniendo resultados muy positivos con estudiantes con TDAH y sin esta en aulas inclusivas. Este sistema de intervención incluye la creación de dos modelos de entrenamiento cognitivo de niños y niñas con hiperactividad o sin esta para actividades orales y escritas en inglés como segunda lengua, ambos basados en Orjales Yillar (2005).

Uno de los mayores problemas que hay en las aulas de Educación Primaria es que los grupos de aprendices no leen correctamente los enunciados. Esto hace que cometan muchos errores o, en el caso de la lengua inglesa, no sepan qué es lo que tienen que hacer. El esquema de autoinstrucciones para el entrenamiento cognitivo de niños y niñas con TDAH y sin esta, para actividades escritas (Figura 2) presentado en el presente artículo, ayuda a al alumnado a prestar atención a la lectura. Conseguimos que los alumnos y las alumnas leyeran meticulosamente los enunciados, empleando el diccionario para comprender las palabras nuevas. El resultado fue muy efectivo, pues prácticamente la totalidad comprendía los enunciados siguiendo estos pasos. También pusimos en práctica este esquema para que revisaran sus propias respuestas, y se obtuvo que muchas veces eran capaces de autocorregirse al revisar de nuevo su tarea.

Con respecto a la comprensión oral, muchos niños y niñas con TDAH tienen grandes dificultades para seguir un discurso oral. Los problemas de concentración que poseen algunos de estos individuos les impide centrar la atención durante un largo período de tiempo. Esto, sumado a la falta de comprensión de muchos vocablos de la lengua inglesa, hacía que gran cantidad de discentes apenas prestara atención a los discursos orales, independientemente de su extensión. Tras poner en práctica el esquema de autoinstrucciones para el entrenamiento cognitivo para actividades orales (Figura 3) el alumnado con dificultades de atención mejoraron, considerablemente, su comprensión. Prestar especial atención a estos alumnos y alumnas, y establecer un sistema de retroalimentación a lo largo del discurso produjo buenos resultados.

Durante los tres años que duró el estudio, trabajamos con varios discentes con TDAH, con diversas características y necesidades. No obstante, pudimos comprobar que todo el grupo se benefició de las estrategias establecidas y de los esquemas de autoinstrucciones creados para el entrenamiento cognitivo de niños y niñas con TDAH o sin esta para actividades escritas y orales. Su nivel de comprensión oral y escrita mejoró considerablemente. En lo concerniente a las producciones orales y escritas, debemos decir que la gran mayoría avanzaron en sus 
composiciones escritas y se sentían con más tranquilidad a la hora de participar oralmente. Aún así, sus problemas de organización estaban patentes y necesitaban ayuda para producir textos.

A lo largo del primer año, se trabajaron las simplificaciones de actividades y se incrementaron las actividades de metodología TPR (Total Physical Response). Como hemos mencionado anteriormente, este tipo de actividades no solo resultan muy atractivas para todo el grupo de aprendices, especialmente para quienes tienen TDAH, sino que también reducen el estrés a la hora de aprender, dado su alto componente lúdico y la permisividad de los movimientos. El alumnado con TDA/TDAH respondió muy bien a los nuevos procedimientos. Algunos casos requerían también de contratos o refuerzos positivos (pegatina, sonrisa en el cuaderno, etc.). Con algunos escolares bastó con la obtención de un refuerzo positivo solo durante un pequeño periodo (periodo de adaptación), para luego considerar su propio aprendizaje como refuerzo o premio. A medida que el alumnado ganaba confianza y seguridad en sí mismo, se retiraban los refuerzos.

El trabajo que se realizó con el grupo de estudiantes sobre el autoconcepto y la confianza en sí marcó una gran diferencia en el proceso de aprendizaje y en los resultados obtenidos por el alumnado con TDA/TDAH en el área de inglés en la presente investigación, ya que algunos de estos niños y niñas tienen afectada su autoestima, bien por los comentarios negativos recibidos, o por la sensación de fracaso que poseen por cometer más errores o necesitar más tiempo de respuesta. A medida que los niños y niñas ganaban confianza en sí, sus producciones mejoraban, lo que hacía que se esforzaran más, pues tomaban consciencia de sus logros. Lo mismo sucedió al incluir técnicas de relajación en las sesiones, los conflictos en el aula se vieron reducidos y la vuelta después del recreo fue más tranquila, lo que repercutió positivamente en las sesiones de lengua inglesa. Dedicar unos minutos de relajación al inicio de las sesiones o en el momento en el que surge algún imprevisto nos permitirá resolver la desavenencia y retomar la clase de nuevo. Y, sobre todo, es muy importante ser conscientes de que el personal docente es transmisor, no solo de conocimientos, sino también de sensaciones, de valores y de rutinas.

Otra observación destacable del estudio es el alto grado de comorbilidad que los individuos con TDAH tienen, puesto que varios alumnos y alumnas con los que se ha trabajado lo presentan, junto con otras alteraciones (p. ej.: trastorno del aprendizaje o problemas con el lenguaje). Ello debe tenerse en cuenta a la hora de planificar y realizar las actividades, pues gran cantidad de estudiantes de estos grupos no son capaces de comprender todas las indicaciones en un segundo idioma o no poseen todavía las destrezas y habilidades necesarias para participar en juegos en los que se trabajan aspectos gramaticales específicos. Si no se organiza adecuadamente, en lugar de favorecer a dicho estudiantado, podemos aumentar su frustración. Una forma de evitar que esto suceda puede consistir en trabajar en pequeños grupos o parejas, de este modo un niño o niña con TDA/TDAH se agruparía con un compañero o compañera que no lo presente, evitando así que tenga que exponerse ante el gran grupo en las actividades o juegos que no domine o que pongan en evidencia las habilidades todavía no adquiridas debido al trastorno que posee. 
http://doi.org/10.15359/ree.25-3.20

http://www.una.ac.cr/educare

educare@una.ac.cr

\section{Conclusiones}

A modo de conclusión final, consideramos que sí logramos nuestro objetivo, pues tras analizar nuestra pedagogía y poner en práctica los conocimientos obtenidos tras nuestra investigación, conseguimos poner en práctica las herramientas necesarias para atender a todo nuestro alumnado, con TDAH y sin este, mejorando los resultados obtenidos en el área de lengua extranjera.

A lo largo de este estudio se han analizado y puesto en práctica metodologías diversas y multitud de actividades con el fin de plantear un modelo realista en el paradigma de la enseñanza y aprendizaje del inglés como segunda lengua con alumnos y alumnas con TDAH. Se han creado estrategias de intervención para abordar las dificultades que pueden surgir a la hora de aprender una lengua al trabajar las cuatro habilidades básicas de un idioma (comprensión y expresión oral y comprensión y expresión escrita) y se han ideado dos modelos de entrenamiento cognitivo de niños y niñas con hiperactividad o sin esta para actividades orales y escritas en inglés como segunda lengua. Consideramos que todo ello puede servir de gran ayuda para profesionales que se encuentren en un aula con aprendices con TDAH que presenten algún tipo de dificultad a la hora de aprender inglés como segunda lengua, puesto que estudios vigentes han podido constatar el enriquecimiento que ofrecen la práctica de las estrategias mostradas en el presente artículo, no solo para la niñez que padece déficit de atención (con hiperactividad o sin esta), sino para todos los miembros que integran los grupos con los que se ha trabajado. La realización de actividades lúdicas y motivadoras hacen que todo el alumnado disfrute de las clases de idiomas, independientemente del nivel de cada quien o de las necesidades que presenten. Lo mismo sucede con las metodologías menos convencionales, como es el método TPR, puesto que el aprendizaje basado en acciones no solo les ayuda a recordar mejor las palabras, sino que también les permite reducir el nivel de estrés que les produce la adquisición de una segunda lengua. Consideramos muy acertado el empleo de refuerzos positivos y el trabajo de mejora de la autoconfianza con estudiantes, así como el empleo de técnicas de relajación para resolver conflictos.

Por otro lado, es importante tener en cuenta la ratio de las aulas a la hora de poner en práctica algunas actividades, puesto que como hemos podido constatar en este análisis, trabajar con grupos de 25 estudiantes o más no permite controlar la correcta ejecución de determinadas acciones por parte de todo el alumnado, como por ejemplo el correcto posicionamiento de los labios al realizar juegos de desarrollo del lenguaje. Consideramos reseñable apuntar la necesidad de que sea un par de profesionales quienes se encarguen de ejecutar las actividades o juegos, para poder trabajar correctamente con un grupo numeroso.

Aunque es fundamental que el profesorado se prepare, recicle y sea capaz de variar su metodología en función de las necesidades de cada aula, de cada individuo o de cada momento, debemos tener en cuenta que la práctica educativa es diversa y que las técnicas 
absolutas no existen. Todos somos diferentes y cada aprendiz tiene sus propias características y necesidades, se pueden cambiar estas a lo largo de su escolarización, sin olvidar el alto grado de comorbilidad que tiene el TDAH. De ahí la importancia de variar las metodologías en función de las características de las aulas, de cada individuo y de cada momento.

Es primordial que la niñez que padece este trastorno sienta que verdaderamente creemos que pueden aprender inglés, pues gran cantidad posee problemas de autoestima, lo que hace que no se crea capaz de adquirir los logros y los conocimientos de sus pares de aula. Es nuestro papel, como profesores y profesoras, hacerles ver que esto no es así. Debemos trabajar objetivos concretos a corto plazo para que vean que sí pueden alcanzarlos y, sobre todo, respetar sus ritmos de aprendizaje. Darles confianza y tiempo es la clave para que crean en sí y se reenganchen a las clases.

\section{Declaración de Material complementario}

Este artículo tiene disponible, como material complementario:

-La versión preprint del artículo en https://doi.org/10.5281/zenodo.4081587

\section{Referencias}

American Psychiatric Association. (2013). Diagnostic and statistical manual of mental disorders DSM-5 (5. ${ }^{\mathrm{a}}$ ed.). American Psychiatric Association Publishing.

Barkley, R. A. (Ed.). (1990). Attention déficit hyperactivity disorder: A handbook for diagnosis and treatment. The Guilford Press.

Barkley, R. A. (2013). Taking charge of ADHD. The complete, authoritative guide for parents (3. ${ }^{\mathrm{a}}$ ed.). The Guilford Press.

Brouwer Konyndyk, I. (2011). Foreign languages for everyone. How I learned to teach second languages to students with learning disabilities. Edenridge Press.

Cook, G. (1989). Discourse. Language teaching. A scheme for teacher education. Oxford University Press.

Cooper-Kahn J. y Foster, M. (2013). Boosting executive skills in the classroom. A practical guide for educators. Jossey-Bass.

Ivanov, S. (2009) Discourse Analysis in EFL reading. Malmö högskola. http://dspace.mah.se/ bitstream/handle/2043/8453/Sergej\%20lvanov\%20degree\%20project.pdf?sequenc 
http://doi.org/10.15359/ree.25-3.20

http://www.una.ac.cr/educare

educare@una.ac.cr

Jefatura del Estado. (10 de diciembre de 2013). Ley orgánica 8/2013, de 9 de diciembre, para la mejora de la calidad educativa. Boletín Oficial del Estado, núm. 295. https://www.boe.es/ buscar/pdf/2013/BOE-A-2013-12886-consolidado.pdf

Lengel, T.y Kuczala, M. (2010): The kinesthetic classroom. Teaching and learning through movement. Corwin Press.

Lezberg, A. y Hilferty, A. (1978). Discourse analysis in the reading class. TESOL Quarterly, 12(1), 47-55. https://doi.org/10.2307/3585790

McCarthy, M. (1991). Discourse analysis for language teachers. Cambridge University Press.

Monschein, M. (2008). The 50 best games for speech \& language development. Hinton House Publishers.

Orjales Yillar, I. (2005). Trastorno por déficit de atención / hiperatividad. 3 TDAH. Estrategias para la mejora de la conducta y el rendimiento escolar del niño con TDAH en el aula. Esquema de Comunicación.

Quintero Gutiérrez del Álamo, F. J. y García Campos, N. (2019). Actualización en el manejo del TDAH. En AEPap (Ed.), Congreso de Actualización Pediatría 2019 (pp. 29-36). Lúa Ediciones 3.0. https://www.aepap.org/sites/default/files/pags. 29-36 actualizacion en el manejo del tdah.pdf

Rief, S. F. (2005). How to reach and teach children with ADD/ADHD. Practical Techniques, strategies, and interventions (2. ${ }^{\text {a }}$ ed.). Jossey-Bass.

Rinvolucri, M. y Davis, P. (1995). More grammar games: Cognitive, affective and movement activities for EFL students. Cambridge University Press.

Rymes, B. (2016). Classroom discourse analysis. A tool for critical reflection (2. ${ }^{\mathrm{a}}$ ed.). Routledge.

Vetter, A., Schieble, M. y Meacham, M. (2018). Critical conversations in english education: Discursive strategies for examining how teacher and student identities shape classroom discourse. National Council of Teachers of English, 50(3), 255-282. http://www.ncte.org/ library/NCTEFiles/Resources/Journals/EE/0503-april2018/EE0503Critical.pdf 